\title{
PREOPERATIVE DECOMPRESSION IN TREATMENT OF LARGE CYSTIC LESIONS OF THE JAWS
}

\author{
Hristo Daskalov', Petko Petrov², Tsvetan Tonchev ${ }^{3}$ \\ ${ }^{1}$ Department of Oral Surgery, Faculty of Dental Medicine, \\ Medical University - Plovdiv, ${ }^{2}$ Departement of Maxillofacial surgery, \\ Faculty of Dental Medicine, Medical University - Plovdiv \\ ${ }^{3}$ Department of Oral and Maxillofacial Surgery and Special Imaging Diagnostics, \\ Medical University of Varna
}

\begin{abstract}
The cysts of the jaws are a common pathological process in the human organism.

The aim of this study is to discuss the perspective effect of the treatment of large mandibular cysts, in which we have applied decompression as the first stage of their operative treatment.

MATERIALS AND METHODS: Subjects of the study are 11 patients with large cystic lesions of the mandible. We used the decompression method as the first stage of their operative treatment.

RESULTS: The effect of the decompression was monitored radiologically. Changes in the size of the lesion and the bone density were noticed.
\end{abstract}

Keywords: jaw cysts, treatment, decompression, bone density

\section{INTRODUCTION}

The cysts of the jaws are a common pathological process in the human organism. They may result from different factors, such as impairment of the embryonic development and genetic predisposition, odontogenic inflammation process, etc.

The cysts grow slowly and expansively. The reason for this is the increased pressure of the cystic fluid, glycosaminoglycans and prostaglandins in the wall of the cyst, activation of enzyme systems, the role of RANKL, etc. $(7,10)$. All these factors cause

Address for correspondence:

Hristo Daskalov

Department of Maxillofacial Surgery,

Faculty of Dental Medicine,

Medical University - Plovdiv

66 Peshtersko Shose Str.

4000 Plovdiv

e-mail: hdaskalov@hotmail.com

Received: December 29, 2015

Accepted: February 16, 2015 bone resorption and an increase of the cyst size, as well as large postoperative bone defects. Their regeneration is often incomplete, and they are filled up with bone and fibrous tissue. Sometimes they do not regenerate, and are filled up only with fibrous tissue. In this case the affected area of the jaw has decreased bone density, size and volume $(2,4)$.

The treatment of jaw cysts is operative. Difficulties are observed in surgeries of large cysts communicating with the mandibular canal, the maxillary sinus and the nasal cavity. In cases of extreme large cysts there is a risk of fracture during or after the surgery (8).

Decompression is a therapeutic method, which involves perforation of the cystic cavity (1). Different types of tubes, acrylic obturators, etc., are used to keep the communication open. The cystic cavity is washed through the opening, which removes the mediators responsible for the growth of the cyst, and reduces the pressure in the cavity. As a result, there is bone regeneration and decrease of the cyst, which fa-

Scripta Scientifica Medicinae Dentalis, vol. 2, No 1, 2016, pp. 39-42 
cilitates the therapy and reduces the risk of postoperative complications $(5,9)$.

The aim of this study is to discuss the perspective effect of the treatment of large mandibular cysts, in which we have applied decompression as the first stage of their operative treatment.

\section{MATERIALS AND METHODS}

Subjects of the study are 11 patients ( 6 men, 5 women), aged 27 to 54; with large cystic lesions of the mandible. Inclusion criteria: large diameter of the cyst, $>4 \mathrm{~cm}$, radiological test (OPG, CT or 3D) (Fig. 1, Fig. 2).

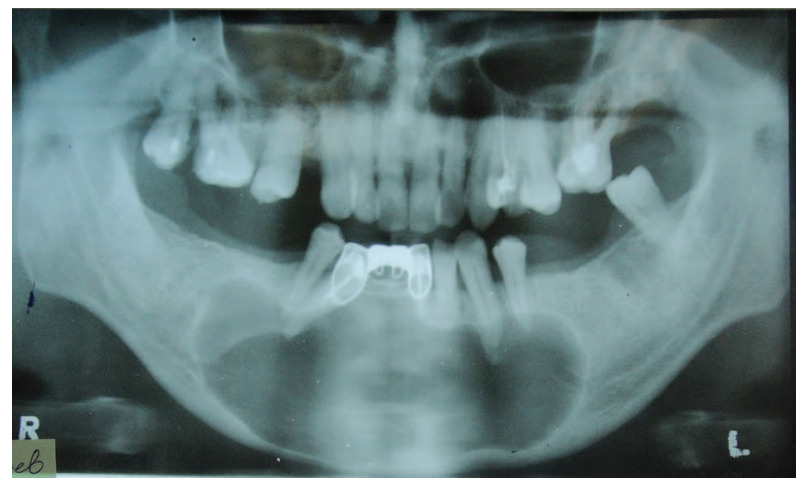

Fig. 1. Cystic lesions of the mandible

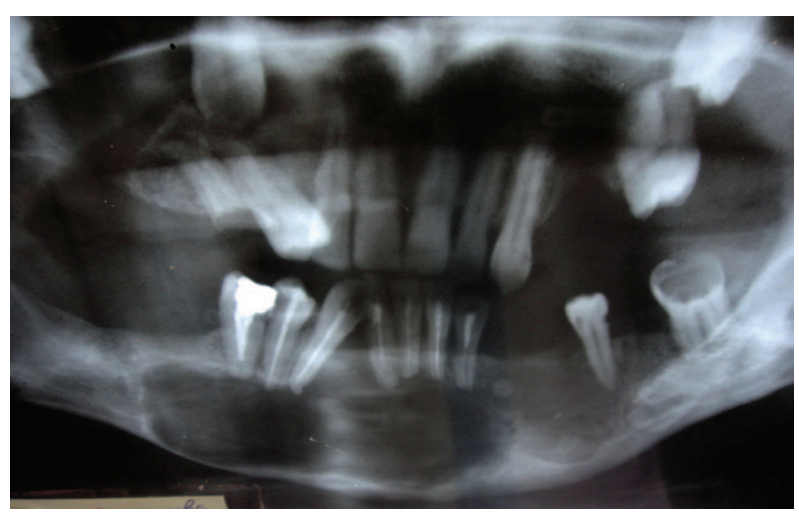

Fig. 2. Cystic lesions of the mandible

\section{Operative Protocol}

In all patients, due to the large size of the lesion, there was a risk of pathological fracture during the surgery, or resection of the jaw was necessary. This motivated our decision to use decompression before the enucleation of the cyst. The surgeries were performed under local or general anesthesia. The vestibular surface of the lesion was perforated and material was taken for a histology test. Depending on the location of the process, we have used an appropriate device to keep the communication open (Fig. 3). The cavity was washed with antiseptic solutions.

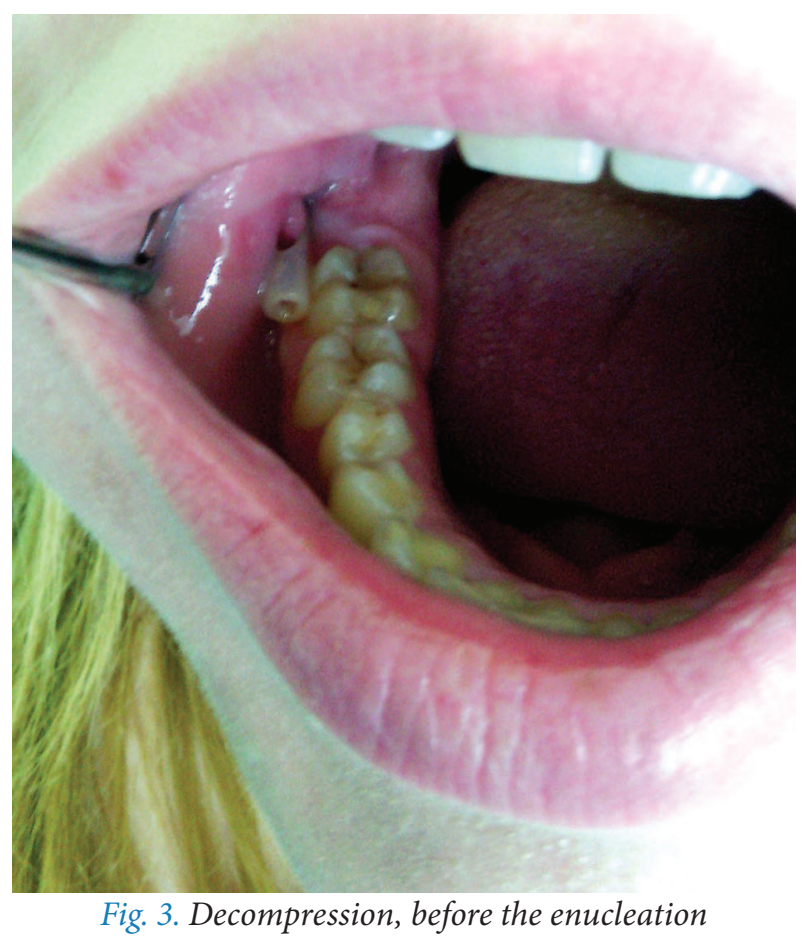

Follow-up and Evaluation of the Reduction of the Lesion

The patients were followed clinically and radiologically for a period of one year. During the clinical observation we noticed sings of exacerbation of the process. The radiological follow-up was done with orthopantomography (OPG). The cystic lesion is not contrast in x-ray test, which allowed us to measure the changes in its size and the bone density. To avoid the differences between the separate OPGs, and to objectify the obtained results, all measurements were done in comparison to the sizes and the density of selected referent objects and zones. The sizes of the lesions and the structure of the new bone before the decompression, and at the end of the observation period, were compared immediately before the extirpation of the cyst.

\section{RESULTS}

\section{Clinical Results:}

In all 11 patients the healing process was without complications. During the whole observation 
process there were no exacerbations or other inflammatory events around the decompression opening.

\section{Cyst Size:}

The reduction of the size was observed every three months with OPG. The reduction rate was different. In one of the cases we observed regeneration of the bone density and no other surgery was necessary (Fig. 4, Fig. 5).

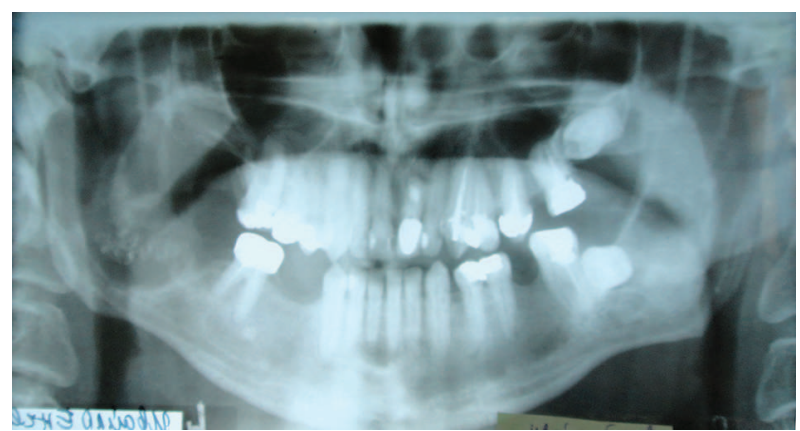

Fig. 4. Before decompression

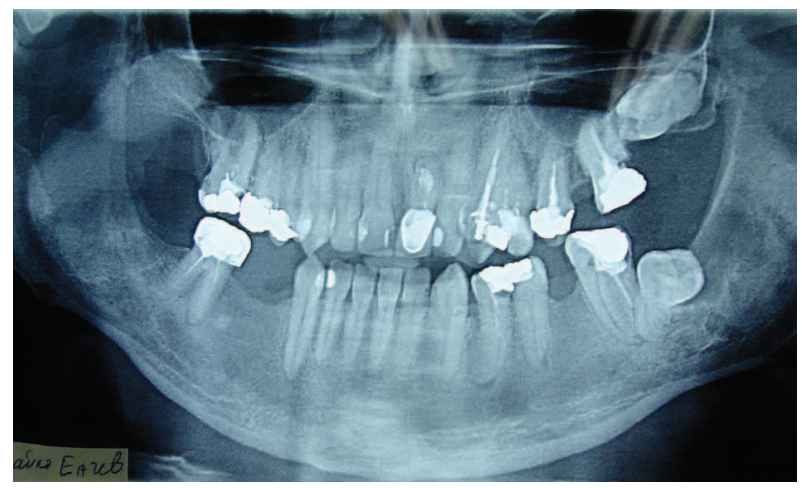

Fig. 5. Result after six months

\section{Bone Density:}

The postoperative $\mathrm{x}$-rays showed an increase of the radiological density in the reduced areas of the cystic lesion during the first 6 months of the observation period. This is an indirect sign of osteogenesis.

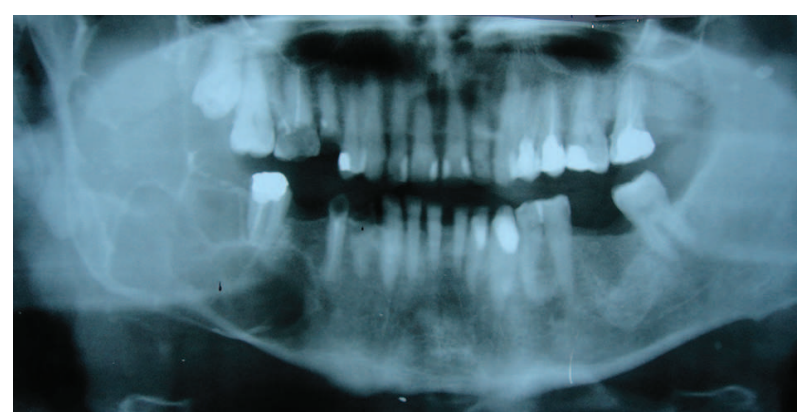

Fig. 6. Before decompression
The radiological results show that 9-12 months after the decompression there is bone formation in all cases (Fig. 6, Fig. 7, Fig. 8).

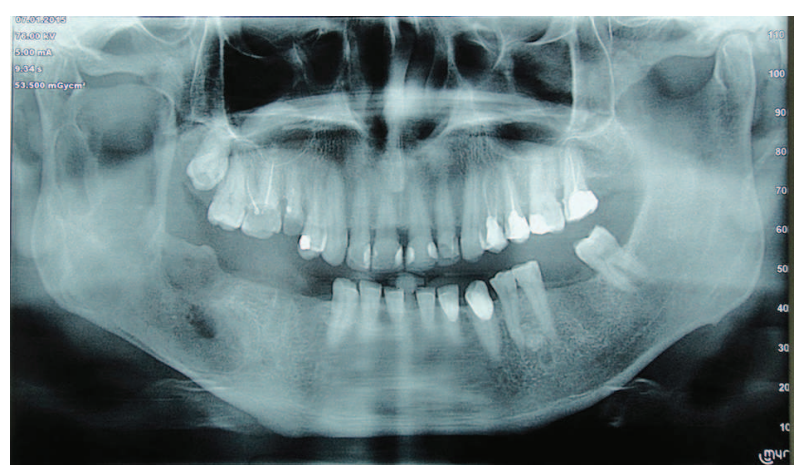

Fig. 7. Result after six months

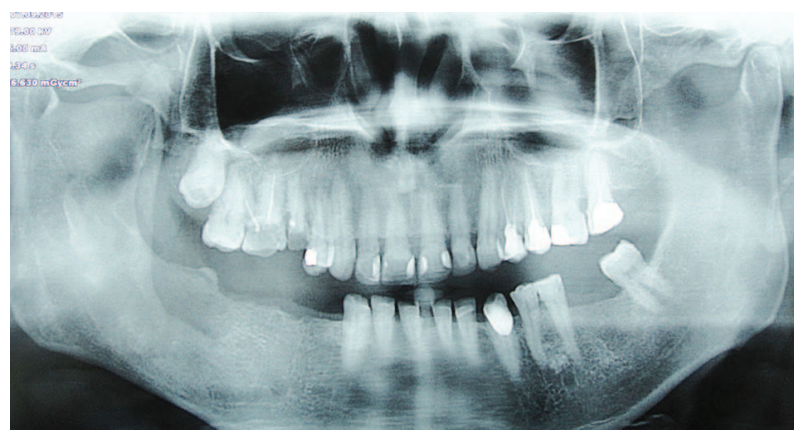

Fig. 8. Result after one year

\section{DISCUSSION}

The most common radiological method for evaluation of the pathological processes in the mandible, and their treatment, is the OPG. In the last years there have been different publications on the spontaneous osteogenesis of maxillary and mandibular bone defects. Decompression is one of the methods for treating odontogenic cystic lesions of the jaws. The advantages of this method are that the therapy starts simultaneously with the collection of information about the type of the cyst. The decompression reduces the size of the cyst and decreases the risks during the surgery. The possibility for pathological or jaw fractures during the extirpation is greatly reduced. At the same time the risk of complications is low.

\section{CONCLUSION}

This study includes limited number of patients. The obtained results allow us to conclude that de- 
compression is an effective method for reduction of the size of the cyst and an increase of the bone density. Regardless of the length of the treatment, we recommend the use of decompression as a stage in the treatment of mandibular cysts.

\section{REFERENCES}

1. Cakarer S, Selvi F, Isler SC, Keskin C. Decompression, enucleation, and implant placement in the management of a large dentigerous cyst. J Craniofac Surg. 2011, 22, 922-4.

2. Carrera M, Dantas D, Marchionni A. Conservative treatment of the dentigerous cyst: report of two cases. Braz J Oral Sci. 2013,12, 1.

3. Chiapasco M, Rossi A, Motta JJ, Crescentini M. Spontaneous bone regeneration after enucleation of large mandibular cysts: A radiographic computed analysis of 27 consecutive cases. J Oral Maxillofac Surg 2000; 58: 942-948.

4. Enislidis G, Fock N, Sulzbacher I, Ewers R. Conservative treatment of large cystic lesions of the mandible: a prospective study of the effect of decompression. Br J Oral Maxillofac Surg. 2004, 42, 546-50.

5. Gao L, Wang L, Li M. Decompression as a treatment for odontogenic cystic lesions of the jaw. J Oral Maxillofac Surg. 2014, 72, 327-33.

6. Jung E, Baek J, Leem D. Decompression Device Using a Stainless Steel Tube and Wire for Treatment of Odontogenic Cystic Lesions: A Technical Report. Maxillofac Plast Reconstr Surg. 2014, 36, 308-310.

7. Hadziabdic N, Sulejmanagic H, Selimovic E, Sulejmanagic N. Therapeutic Approach to Large Jaw Cysts. HealthMED. 5, 2011.

8. Motamedi M, Talesh K. Management of extensive dentigerous cysts. British Dental Journal. 2005, 198, $203-206$.

9. Park H, Song I, Seo B. The effectiveness of decompression for patients with dentigerous cysts, keratocystic odontogenic tumors, and unicystic ameloblastoma. Korean Assoc Oral Maxillofac Surg. 2014, 40, 260-265.

10. Pechalova PF, Bakardjiev AG, Beltcheva AB. Jaw cysts at children and adolescence: A single-center retrospective study of 152 cases in southern Bulgaria. Med Oral Patol Oral Cir Bucal. 2011 Jan 10. 